\title{
SUMMARY OF THE WORKING GROUP ON THE CONSTRUCTION AND DEMONSTRATION OF A SIBERIAN SNAKE*
}

L.G. Ratner, Coordinator

Brookhaven National Laboratory, Upton, NY 11973

R.R. Raylman, Scientific Secretary

Randall Laboratory of Physics, University of Michigan Ann Arbor, MI 48109-1120

It was recognized as long ago as the 1977 Ann Arbor Meeting on Higher Energy Polarized Proton Beams" that a "Siberian Snake" should be built and tested in an operating accelerator. A program to accomplish this at Argonne National Laboratory (ANL) was part of a proposal to DOE to construct a small R\&D accelerator at ANL. This was never implemented and between then and now many theory papers have been written but no Proof of Principle Experiment ( $P O P E$ ) has been done. The present Ann Arbor workshop again strongly recommends that a POPE be implemented as soon as possible since it has important consequences for all of the new higher energy machines.

This "Demo Snake" work1ng group zeroed in on the Alternating Gradient Synchrotron (AGS) as the place where such a project could now be most effectively done. The AGS has a polarized beam and knowledgeable theoret 1 cal and experimental accelerator physlcists who have been working with polarized beams in accelerators for the last few years. Referring to the information contained in the introductory remarks to the working group (see preceding paper) on the characteristics of snakes, we can detail the necessary design for the AGS.

A. It must be a solenoid and not transverse field magnets.

1. Transverse field magnets require spaces longer than the AGS 3M straight-section.

2. Polarized beam must be injected (200 MeV) and this low energy makes impractically large apertures necessary even if one could make shorter ultra high field magnets.

B. Given a solenoid,

1. It must precess the spin by $\theta=\pi$ and this requires $37.5 \mathrm{KG}-\mathrm{M}$ per Gev/c.

2. The AGS strong resonances $G \gamma=0+\nu$ and $G \gamma=9$ occur just below $5 \mathrm{Gev} / \mathrm{c}$.

3. We therefore need $187.5 \mathrm{KG}-\mathrm{M}$ and considering $3 \mathrm{M}$ space available we should design a $2 \mathrm{M} 94 \mathrm{KG}$ solenoid.

4. It must track the accelerator field from injection $(644 \mathrm{MeV} / \mathrm{c})$ to the desired $5 \mathrm{Gev} / \mathrm{c}$.

*Work performed under the auspices of the U.S. Department of Energy. 
5. Since we need a superconducting solenoid to reach the desired field, we will probably operate the AGS with a slower rep rate to give a few seconds to ramp up the solenoid.

6. Since this is a test of snake characteristics, we can make the solenoid aperture small, say $5 \mathrm{~cm}$ even though beam intensity will suffer.

Solenoid is then: $2 \mathrm{M}$ long $\times 5 \mathrm{~cm}$ bore at $94 \mathrm{KG}$ with a rise time of, say, 4 sec. to $5 \mathrm{Gev} / \mathrm{c}$.

This solenoid is a very strong focussing device and will require corrections to be made in order to accelerate beam. Quadrupoles and skew quadrupoles will be necessary to compensate for the effect of the solenoid. Computer simulations will have to be done to determine the strength and placement of correcting elements. It will be almost impossible to get much more than the $2 \mathrm{M}$ solenoid into a $3 \mathrm{M}$ straight-section. It would be very advantageous if we could correct globally by using the quadrupoles and skew quadrupoles already in the AGS.

Assuming the satisfactory construction, installation, and correction for the solenoid, we can now outline the various tests which appear desirable.

Besides the question of the efficacy of the solenoid snake there is the question of adiabatic turn-on. We would propose to first test the snake by injecting with the snake set to the appropriate field and then ramping to match the acceleration cycle and then investigate adiabatic turn-on.

The solenoid snake requires a beam polarized in the horizontal plane to give a stable non-depolarizing condition. This can be done in two ways. One method involves taking the protons out of the source polarized longitudinally and inject into the AGS with longitudinal polarization. The other method would transport the beam vertically polarized from the source and precess it by $\pi / 2$ into the horizontal plane with a $12.1 \mathrm{KG}-\mathrm{M}$ solenofd. The spin would precess by $\theta_{p}=\gamma G \theta_{B}$ in the AGS. The snake solenoid has to be placed so that the spin is longitudinal at $180^{\circ}$ from it. The polarization must be measured where the spin is transverse, so a polarimeter would have to be placed at $\theta_{p}=\pi / 2$ from the solenoid. The first test then involves infecting the beam at $200 \mathrm{MeV}$ and ramping the solenold from $24 \mathrm{KG}-\mathrm{M}$ to $187 \mathrm{KG}-\mathrm{M}$ as the beam is being accelerated to $5 \mathrm{Gev} / \mathrm{c}$. The accelerator and solenoid will be flattopped and the polarization measured at $5 \mathrm{Gev} / \mathrm{c}$. The polarimeter must have detectors in the vertical plane to measure the transverse spin. This test would tell us the efficiency of the snake in passing through three strong resonances, $G \gamma=8, G \gamma=0+v, G \gamma=9$.

There are then several tests of adiabatic turn-on which can be done.

1. Inject a beam with vertical spin and accelerate to $5 \mathrm{Gev} / \mathrm{c}$ flat-top and then adiabatically turn on the solenoid. This should flip the spin into the horizontal plane. The vertical polarization should go to zero and the transverse 
should go to maximum. One can also turn the solenold on and off to see if the vertical and transverse spin conditions follow.

2. The turn on and off may proceed more efficiently if the condition $K=G \gamma=n+1 / 2$ (SPIN TUNE) exists on the flat-top. For the AGS $K=9-1 / 2$ at $4.88 \mathrm{Gev} / \mathrm{c}$ and we can set the flat-top at that value. One could also consider injecting the beam, accelerating from $200 \mathrm{MeV}$ to a 370 $\mathrm{MeV}$ flat-top where $\kappa=2-1 / 2$ and then turning on the solenold. Then one would ramp the accelerator and the solenoid to $4.88 \mathrm{Gev} / \mathrm{c}$.

3. This test would inject the beam with vertical polarization and ramp the solenoid and the accelerator from injection to flat-top and again investigate the beam on flattop.

4. In conjunction with adiabatic turn-on the idea arose that the snake might work for a range of precession values and not necessarily only for $\pi$. To test this we might continue to accelerate to $9.64 \mathrm{Gev} / \mathrm{c}$ where $K=18-1 / 2$ and $\theta_{p} \approx \pi / 2$.

In conclusion, it appears that a solenoid snake of reasonable dimensions can be tried in the AGS and could answer the questions of whether such devices will make possible polarized beams at very high energies.

\section{REFERENCE}

1. A.D. Krisch, A.J. Salthouse, Editors, AIP Conference Proceedings No. 12 High Energy Polarized Proton Beams, Ann Arbor, 1977.

\section{WORKING GROUP}

$\begin{array}{lll}\text { Full time: } & \text { L. Ratner (Coord.) } & \text { J. Norem } \\ & \text { G. Danby } & \text { D. Peaslee } \\ \text { Y.Y. Lee } & \text { R. Raylman } \\ \text { Y. Makdisi } & \\ \text { Contributions from: } & \text { J. Buon } & \text { G. Phillips } \\ & \text { A. Chao } & \text { R. Ruth } \\ & \text { E. Courant } & \text { K. Steffen } \\ & \text { A. Krisch } & \text { L. Teng } \\ \text { S. Lee } & \text { S. Tepikian } \\ & \text { B. Montague } & \text { K. Terwilliger }\end{array}$

